\title{
High-Resolution Transmission Electron Microscopy With Zach Phase Plate
}

\author{
Simon Hettler ${ }^{1}$, Manuel Dries ${ }^{1}$, Tina Schulze ${ }^{1}$, Marco Oster $^{2}$, Christian Wacker ${ }^{2}$, Rasmus R. Schröder $^{2}$ \\ and Dagmar Gerthsen ${ }^{1}$ \\ 1. Karlsruher Institut für Technologie, Laboratorium für Elektronenmikroskopie, Karlsruhe, Germany. \\ 2. BioQuant CellNetworks, Universität Heidelberg, INF 267, D-69120 Heidelberg, Germany.
}

Physical phase plates (PP) for transmission electron microscopy (TEM) have gained tremendous attention in the past decade. PPs introduce a relative phase shift between scattered and unscattered electrons and thus enhance the contrast of weak-phase objects in TEM. Several approaches to PP TEM exist [1] with thin-film PPs being the most frequently used concept up to now [2]. A major disadvantage of thin-film PPs is scattering of electrons by the PP itself which induces a damping of the phase contrast transfer and reduces the resolution of the microscope. The electrostatic Zach-PP uses a strongly localized electrostatic potential to induce the phase shift and is not affected by this effect [3]. In this work, the application of a Zach-PP for high-resolution (HR) TEM is investigated by studying a crystalline Si-sample.

The Zach-PP (Fig. 1) consists of a single rod with an Au electrode which is surrounded by insulating and shielding electrically conductive layers. If a voltage is applied to the electrode, an inhomogeneous potential emerges at the PP tip. The PP was implemented in the back-focal plane of a Zeiss $923 \Omega$ transmission electron microscope equipped with a field-emission gun operated at $200 \mathrm{kV}$ and a $4 \mathrm{k}$ TVIPS CCD-camera. For PP TEM imaging, the tip is usually positioned close to the zero-order beam to minimize the cut-on frequency and enhance the contrast of weak-phase objects up to $10 \mathrm{~nm}$ size [4]. For HR PP TEM in this work, the cut-on frequency is of minor importance and the PP was positioned at an increased distance to the zero-order beam to reduce charging effects.

Fig. 2 presents results obtained with a single-crystalline Si-sample in [110] zone-axis orientation. Fig. 2a shows a HR PP TEM image in an overview perspective which was acquired without applied voltage. A relatively thick amorphous region is observed which results from Si-oxide formation and amorphization during sample preparation. The marked area is displayed enlarged in Fig. 2b where (111)-type lattice fringes are clearly resolved. The power spectrum of the HR PP TEM image (Fig. 2a) is shown in Fig. 2c and reveals the four (111)-type and the (002)-reflections as well as the PP rod. The influence of the phase shift on the reflection intensities is analyzed by acquiring an image series with varying the PP voltage from $-5 \mathrm{~V}$ to $+5 \mathrm{~V}$. The reflection intensity of the (111)-reflections in the corresponding power spectra is plotted in Fig. 2d, where the blue and red crosses are the averaged intensities from the reflections marked in Fig. 2c. The data is well fitted by a function $\sim\left|\cos \left(\varphi_{P P}+\theta\right)\right|$ as predicted by theory. The phase $\theta$ comprises the wave aberration function and the phases of the zero-order beam and the corresponding (111)-type reflection. The results show, that the electrostatic Zach-PP does not reduce the resolution of the microscope and is well suited for HR TEM applications [5].

References:

[1] R M Glaeser, Rev. Sci. Instrum. 84 (2013), 111101.

[2] R Danev, K Nagayama, Ultramicroscopy 88 (2001), p. 243-252.

[3] K Schultheiss et al, Microsc. Microanal. 16 (2010), p. 785-794.

[4] N Frindt et al, Microsc. Microanal. 20 (2014), p. 175-183.

[5] Financial support by German Research Foundation (DFG) under contract Ge 841/16 and Sch 424/11. 


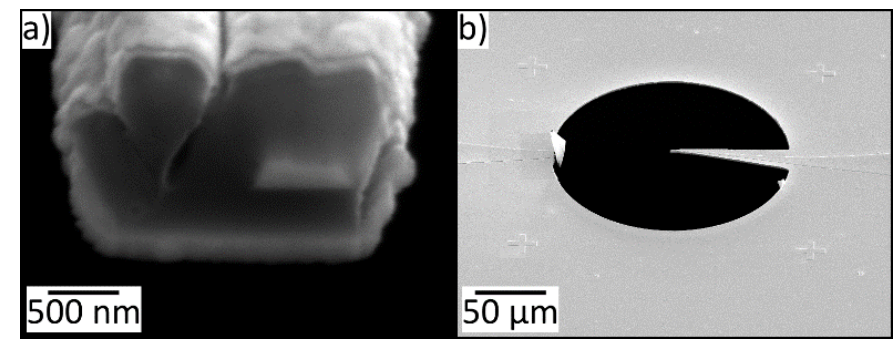

Figure 1. Scanning electron microscopy images of the Zach PP used in the experiments. (a) Image of the PP tip shows the layer system of central electrode, surrounding insulating layers and shielding metal layers. (b) The PP aperture has a diameter of $90 \mu \mathrm{m}$.

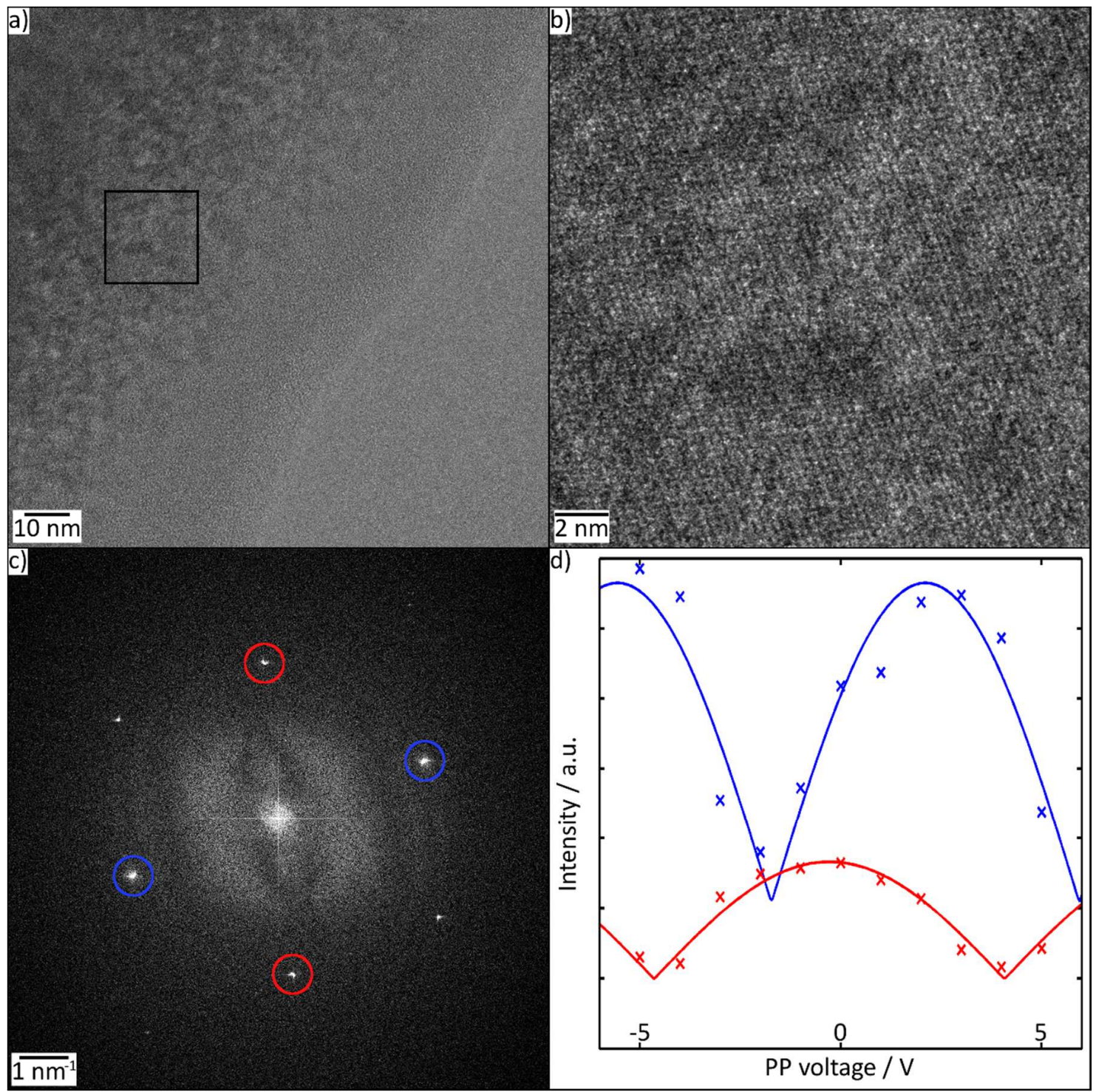

Figure 2. HR PP TEM images and analysis. (a) Overview HR PP TEM image of the Si-sample in [110] zone-axis orientation. (b) Detail image of the area marked by a black frame in (a). (c) Power spectrum of the image in (a) with (002)-reflections and (111)-type reflections marked by the red and blue circles. (d) Plot of the averaged reflection intensity of the four (111)-type reflections marked blue and red in (c) as a function of the applied voltage. Solid lines represent fits with a $\left|\cos \left(\varphi_{P P}+\theta\right)\right|$ behavior. 